This item was submitted to Loughborough's Research Repository by the author.

Items in Figshare are protected by copyright, with all rights reserved, unless otherwise indicated.

\title{
Nonsmooth adaptive control for uncertain nonlinear systems: a non-recursive design approach
}

PLEASE CITE THE PUBLISHED VERSION

https://doi.org/10.1109/lcsys.2021.3065187

\section{PUBLISHER}

Institute of Electrical and Electronics Engineers (IEEE)

VERSION

AM (Accepted Manuscript)

\section{PUBLISHER STATEMENT}

(c) 2021 IEEE. Personal use of this material is permitted. Permission from IEEE must be obtained for all other uses, in any current or future media, including reprinting/republishing this material for advertising or promotional purposes, creating new collective works, for resale or redistribution to servers or lists, or reuse of any copyrighted component of this work in other works.

\section{LICENCE}

\section{All Rights Reserved}

\section{REPOSITORY RECORD}

Zhang, Chuanlin, and Jun Yang. 2021. "Nonsmooth Adaptive Control for Uncertain Nonlinear Systems: A Non-recursive Design Approach”. Loughborough University. https://hdl.handle.net/2134/16578584.v1. 


\title{
Nonsmooth adaptive control for uncertain nonlinear systems: a non-recursive design approach
}

\author{
Chuanlin Zhang, Senior Member, IEEE and Jun Yang, Senior Member, IEEE
}

\begin{abstract}
In this paper, a one-step nonsmooth adaptive controller design framework is proposed the first time for a class of nonlinear systems with general non-parametric uncertainties. By virtue of a novel non-recursive synthesis philosophy, a nonsmooth adaptive stabilizer can be constructed straightforwardly from the system in a possible simplest form, which facilitates practical implementations. In reference to well-acknowledged recursive design based approaches, direct improvements with this new nonrecursive methodology include largely reduced synthesis complexity along with an essential detachment of control law design and stability analysis. A numerical example is provided to demonstrate both the simplicity and effectiveness of the proposed algorithm.
\end{abstract}

Index Terms-adaptive stabilization, non-recursive design, homogeneous system theory, non-parametric uncertainty

\section{INTRODUCTION}

$\mathbf{S}$ INCE the era of modern control theory, robust and adaptive control strategies have been well established for systems in the presence of uncertain parameters, unknown actuator nonlinearities, unmodelled dynamics and external disturbances, etc. Various control strategies can be found in the literature regarding the synthesis issue for uncertain nonlinear systems, such as high-gain control [1], adaptive backstepping control [2], nonsmooth control [3], [4], [5], [6], etc. As one classical design algorithm, adaptive backstepping design approach is well developed under the condition that the system should subject to a parametric structure [2]. However, for nonlinear systems with the presence of non-parametric uncertainties, an alternative synthesis tool is of significance, while fewer results can be found. It is reported in [7], [8], [9], [10], a time-varying scaling gain method can be found, which could derive universal control laws by means of a dynamic high-gain domination technique. However, a Lipschitz continuous condition is required for the assurance of a global asymptotical stabilization result.

By noting that in reference to popular asymptotical control strategies, nonsmooth control design could provide the closedloop system a faster convergence rate and stronger robustness

This work is supported in part by National Natural Science Foundation of China (No. 61503236 and 61973080), in part by the Program for Professor of Special Appointment (Eastern Scholar) at Shanghai Institutions of Higher Learning and in part by Shanghai Rising-Star program (NO. 20QA1404000) (Corresponding author: J. Yang).

Chuanlin Zhang is with the College of Automation Engineering, Shanghai University of Electric Power, Shanghai, China, 200090 (e-mail: clzhang@shiep.edu.cn)

Jun Yang is with the Department of Aeronautical and Automotive Engineering, Loughborough University, Loughborough LE11 3TU, U.K. (e-mail: j.yang3@lboro.ac.uk)
[11], it hence arouses a great deal of interests from the control community in recent decades. By incorporating an adding a power integrator approach, a systematic robust nonsmooth control design methodology has been well developed, see e.gs., [12], [13] and references therein. Regarding the finite-time adaptive stabilization design, under a polynomial hypothesis of the system structure, the work in [3] proposes a nonsmooth adaptive design framework while a later result in [4] has extended the finite-time adaptive control issue to a class of $p$-normal uncertain nonlinear systems. In a recent research, [14] has proposed a finite-time stabilizing controller with timevarying gains being tuned online by two dynamic equations. Later on, [15] develops a finite-time stabilization result via the adding a power integrator technique. [16] shows the existence of a monotone dynamic scaling gain, whose explicit construction procedure for practices, however remains open. If an uncertain high order system is considered, a recent study in [17] addresses the adaptive asymptotical stabilization problem by incorporating a dual-layer update law with the adding a power integrator technique. Using a similar dynamic handling strategy, the work [18] develops an adaptive finitetime stabilization result under a modified global nonlinearity restriction hypothesis.

It is worth pointing out here that most of the above mentioned adaptive design approaches involve a recursive determination of virtual controllers. Direct drawbacks may exhibit on the expanding complexity when the system order is growing (also be called as "explosion of complexity"). Meanwhile, the gain selection guideline is made much conservative by going through a variety of necessary mathematical estimations, see for instances, [14], [16], etc. These facts can be regarded as main obstacles for wide application of advanced nonlinear control algorithms in real-life control engineering. In the literature, there are some recent advances focusing on the relaxation of controller design complexity by proposing a one-step or non-recursive design strategy. For instance, by integrating the dynamic surface control with neural networks, a one-step neuroadaptive control scheme is proposed in [19], aiming to avoid the laborious repetitive design procedures. A later result in [20] proposes an adaptive NN output feedback control law for constrained pure-feedback systems in a nonrecursive manner. In [21], a non-recursive adaptive controller is constructed for an uncertain underactuated crane system. A novel $\mathbb{C}^{1}$ adaptive controller is also built in a non-recursive manner in [22], where the cumulative complexity in classical backstepping design can be totally avoided.

Regarding the nonsmooth control case, it is reported in [23] that a global robust homogeneous stabilizer for a class 
of inherent nonlinear systems can be built in a novel nonrecursive synthesis manner. It is illustrated that the control design procedure is largely simplified by neglecting recursive virtual controllers. Later on, based on this initiative, a non-recursive finite-time exact tracking control algorithm for nonlinear systems with mismatched disturbances is proposed in [24]. However, on one hand, these robust design methods with conservatively selected control gain may behave a deteriorate transient-time performance when the system is facing largely varying disturbances/uncertainties. On the other hand, the global non-recursive dynamic control design issue for nonlinear systems with non-parametric uncertainties is not a trivial extension from its robust control counterpart. Firstly, very few existing results can be referred to regarding one-step nonsmooth synthesis approach for nonlinear systems with non-parametric uncertainties. Secondly, the online estimation/identification of the internal coupled uncertainties and its implicit relation with control gain tuning mechanism are still very intricate. Thus, in order to present an explicit and simple gain tuning mechanism, new design tools should be investigated.

Inspired by the design facility of homogeneous system theory as illustrated in the existing literatures partially mentioned above, a non-recursive nonsmooth adaptive controller design framework is proposed the first time to solve the dynamic stabilization problem for a class of nonlinear systems with the presence of general non-parametric uncertainties. To assure the theoretical justifications, rigourous stability analysis is presented by guaranteeing first the non-existence of a finite escape time and then a local finite time convergence rate. Later on, a numerical example and its simulation verification are offered to demonstrate the design simplicity and control performance of the proposed control scheme. In reference to existing related results, the main contributions of this paper can be summarized as the following two aspects:

- As a direct benefit from the non-recursive design approach, a simplest non-nested stabilizing controller can be constructed independent of the Lyapunov function based stability analysis.

- The control gain vector can be easily determined while the dynamic scaling gain is online updated subjecting to an explicit self-tuning adaption law.

The remainder of this paper is organized as follows. Some notations, definitions, useful lemmas and problem statement are listed in Section 2. The main result of this paper is presented in Section 3. In Section 4, a numerical simulation result is provided. Finally, a conclusion and a reference list end the paper.

\section{Preliminaries and Problem Statement}

\section{A. Preliminaries}

The following definitions and notations are provided for briefness of expressions.

i) For integers $j$ and $i$ satisfying $0 \leq j \leq i$, denote $\mathbb{N}_{j: i}:=$ $\{j, j+1, \cdots, i\}$. The symbol $\mathbb{C}^{i}$ denotes the set of all differentiable functions whose first $i$-th time derivatives are continuous. $\mathfrak{R}_{+}$denotes the set of positive real numbers. A continuous function $\lfloor x\rceil^{r}$ with $r \in \mathfrak{R}_{+}$is defined by $\lfloor x\rceil^{r} \triangleq \operatorname{sign}(x)|x|^{r}$. ii) [25] For a coordinate $x \in \mathfrak{R}^{n}$, and positive real numbers $\left(r_{1}, r_{2}, \cdots, r_{n}\right) \triangleq r$, a one-parameter family of dilation is a map $\Delta^{r}: \Re_{+} \times \mathfrak{R}^{n} \rightarrow \mathfrak{R}^{n}$, defined by $\Delta_{\varepsilon}^{r} x=\left(\varepsilon^{r_{1}} x_{1}, \cdots, \varepsilon^{r_{n}} x_{n}\right)$ where $\varepsilon>0$ is a constant parameter. For a given dilation $\Delta^{r}$ and a real number $\tau$, a continuous function $V: \mathfrak{R}^{n} \rightarrow \mathfrak{R}$ is called $\Delta^{r}$-homogeneous of degree $\tau$, denoted by $V \in \mathbb{H}_{\Delta^{r}}^{\tau}$ if $V\left(\Delta_{\mathcal{\varepsilon}}^{r}\right)=$ $\varepsilon^{\tau} V$. A continuous vector field $f(x)$ is $\Delta^{r}$ - homogeneous of degree $\tau$, if $f_{j} \in \mathbb{H}_{\Delta^{r}}^{\tau+r_{j}}, j \in \mathbb{N}_{1: n}$. A homogeneous vector $\lfloor x\rceil_{\Delta^{r}}^{\tau}$ is denoted by $\lfloor x\rceil_{\Delta^{r}}^{\tau} \triangleq \operatorname{col}\left(\left\lfloor x_{1}\right\rceil^{\frac{\tau}{r_{1}}}, \cdots,\left\lfloor x_{n}\right\rceil^{\frac{\tau}{r_{i}}}\right)$. A homogeneous $2-$ norm is defined by $\|x\|_{\Delta^{r}}=\left(\sum_{i=1}^{n}\left|x_{i}\right|^{2 / r_{i}}\right)^{1 / 2}$.

Some useful lemmas are stated as follows for the convenience of readers.

Lemma 2.1: [25] Let $\Phi_{1}(x) \in \mathbb{H}_{\Delta^{\kappa}}^{\tau_{1}}$ and $\Phi_{2}(x) \in \mathbb{H}_{\Delta^{\kappa}}^{\tau_{2}}$, respectively, then the following statements hold

i) $\Phi_{1}(x) \Phi_{2}(x) \in \mathbb{H}_{\Delta^{\kappa}}^{\tau_{1}+\tau_{2}}$.

ii) $\frac{\partial \Phi_{1}(x)}{\partial x_{i}} \in \mathbb{H}_{\Delta^{\kappa}}^{\tau_{1}-r_{i}}, i \in \mathbb{N}_{1: n}$.

iii) If $\Phi_{1}(x), \Phi_{2}(x)$ are positive definite, then there exists two constants $b_{1} \in \mathfrak{R}_{+}$and $b_{2} \in \mathfrak{R}_{+}$, such that $b_{1} \Phi_{1}^{\frac{\tau_{2}}{\tau_{1}}}(x) \leq \Phi_{2}(x) \leq$ $b_{2} \Phi_{1}^{\frac{\tau_{2}}{\tau_{1}}}(x)$.

Lemma 2.2: [11] Consider a dynamical system $\dot{\eta}=$ $f(\eta), f(0)=0$, where $f: D \rightarrow \mathfrak{R}^{n}$ is non-Lipschitz continuous on an open neighborhood $D$ of the origin $\eta=0$ in $\Re^{n}$. If there exist an open neighborhood $U$ of the origin, a $\mathbb{C}^{1}$ positivedefinite and proper Lyapunov function $V: U \backslash\{0\} \rightarrow \mathfrak{R}_{+}$and real numbers $c \in \mathfrak{R}_{+}$and $\imath \in(0,1)$, such that $\dot{V}+c V^{\imath} \leq 0$ for $\eta \in U$, then the origin $\eta=0$ is a locally finite-time stable equilibrium with a settling time $T \leq \frac{V^{1-l}\left(\eta_{0}, t_{0}\right)}{c(1-\imath)}$ for any given initial condition $\eta_{0}=\eta\left(t_{0}\right)$.

Lemma 2.3: Let $D=\operatorname{diag}\left\{d_{1}, d_{2}, \cdots, d_{n}\right\}$ where $d_{i}>0, i \in$ $\mathbb{N}_{1: n}$ is a constant and $A \in \mathfrak{R}^{n \times n}, B \in \mathfrak{R}^{n}$ be matrices in the controllable canonical form. Then there exist a positive definite, symmetrical matrix $P \in \mathfrak{R}^{n \times n}$ and a row vector $K=$ $\left[k_{1}, \cdots, k_{n}\right]$, and two positive constants $v_{1}$ and $v_{2}$, such that $(A-B K)^{\top} P+P(A-B K) \leq-I, v_{1} I \leq D P+P D \leq v_{2} I$.

Proof: The proof follows straightforwardly from Theorem A1 in [7], hence is omitted here.

\section{B. Problem Statement}

This paper revisits a class of lower-triangular nonlinear system with non-parametric uncertainties, depicted by the following form:

$$
\left\{\begin{array}{l}
\dot{x}_{i}=x_{i+1}+f_{i}\left(\theta, \bar{x}_{i}\right), i \in \mathbb{N}_{1: n-1}, \\
\dot{x}_{n}=u+f_{n}(\theta, x)
\end{array}\right.
$$

where $\bar{x}_{i}=\operatorname{col}\left(x_{1}, x_{2}, \cdots, x_{i}\right), x=\bar{x}_{n}$ and $u$ are the system partial state vector, full state vector and control input, respectively, $\theta \in \mathfrak{R}^{r}$ is an unknown bounded parameter vector which could be either time-varying or constant, $f_{i}(\cdot), i \in \mathbb{N}_{1: n}$ is a continuous nonlinear function satisfying a vanishing condition, i.e., $f_{i}(\theta, 0)=0$ for $i \in \mathbb{N}_{1: n}$. Through out this paper, the initial time is set as $t_{0}$ while the initial state vector is denoted by $x_{0} \triangleq x\left(t_{0}\right)$.

The control objective of this paper is to find a dynamic 
stabilizing controller of the following non-nested form:

$$
\left\{\begin{array}{l}
u=u(\ell, x) \in \mathbb{C}^{0}, u(\ell, 0)=0, \\
\dot{\ell}=\psi(\ell, x) \in \mathbb{C}^{1}\left([1,+\infty) \times \mathfrak{R}^{n} \rightarrow[0,+\infty)\right), \\
\ell\left(t_{0}\right)=1,
\end{array}\right.
$$

such that the following conclusions hold for the resulting closed-loop system (1)-(2).

i) All the signals are globally uniformly bounded.

ii) The origin $x=0$ is a finite-time stable equilibrium.

\section{Non-Recursive Adaptive Controller Design}

In this section, three handling procedures are presented to better illustrate the nonsmooth adaptive stabilization design.

Step 1. Guideline for the Selection of Design Parameters

With the denotation that $A, B$ are matrices of controllable canonical form, $\digamma(\theta, x)=\operatorname{col}\left(f_{1}(\cdot), \cdots, f_{n}(\cdot)\right)$, system (1) can be rewritten as

$$
\dot{x}=A x+B u+\digamma(\theta, x) .
$$

Throughout this paper, the homogeneous dilation weight $r$ is set as $r_{1}=1, r_{i}=r_{i-1}+\tau, i \in \mathbb{N}_{2: n}$ with a degree $\tau \in\left(-\frac{1}{n}, 0\right)$. Let $\kappa>1$ be a pre-set design constant. Denote $\kappa-\tau / 2=\gamma$, $\Xi_{1} \triangleq \operatorname{diag}\left\{\frac{\gamma}{r_{1}}, \frac{\gamma}{r_{2}}, \cdots, \frac{\gamma}{r_{n}}\right\}$ and $\Xi_{2} \triangleq \operatorname{diag}\left\{0, \frac{\gamma}{r_{2}}, \cdots, \frac{(n-1) \gamma}{r_{n}}\right\}$.

It is obvious that $\gamma>0$ and $r_{i}>0$. Hence by following Lemma 2.3, there exist a positive definite, symmetrical matrix $P \in \mathfrak{R}^{n \times n}$ and a gain vector $K$, such that

$$
\begin{aligned}
& (A-B K)^{\top} P+P(A-B K) \leq-I, \\
& \Xi_{1} P+P \Xi_{1}>0 .
\end{aligned}
$$

Based on the relation (4), we could define a sufficiently large constant design parameter $\rho$ satisfying $\rho>$ $\max \left\{0,-\frac{\lambda_{\min }\left(\Xi_{2} P+P \Xi_{2}\right)}{\lambda_{\min }\left(\Xi_{1} P+P \Xi_{1}\right)}\right\}$.

Step 2. Change of Coordinates: Define a $x-\xi$ coordination transform by the following form

$$
\xi_{i}=x_{i} / \ell^{\rho+i-1}, i \in \mathbb{N}_{1: n}, v=u / \ell^{\rho+n},
$$

where $\ell$ is a scaling gain function to be made precise by an update law later on. By denoting

$$
\begin{gathered}
\xi=\operatorname{col}\left(\xi_{1}, \cdots, \xi_{n}\right), D=\operatorname{diag}\{0,1, \cdots, n-1\}, \\
\tilde{\digamma}(\ell, \theta, x)=\operatorname{col}\left(f_{1}(\cdot) / \ell^{\rho}, \cdots, f_{n}(\cdot) / \ell^{\rho+n-1}\right),
\end{gathered}
$$

and $I_{n}$ as an identity matrix, it is not difficult to derive that the $\xi$-system dynamics should be expressed as the following compact form:

$$
\dot{\xi}=\ell(A \xi+B v)-\left(\rho I_{n}+D\right) \xi \dot{\ell} / \ell+\tilde{\digamma}(\ell, \theta, x) .
$$

Step 3. Dynamic Control Law Construction: At this stage, corresponding to (2), we are already able to design a dynamic state feedback stabilizing controller of the following simplest form:

$$
\left\{\begin{array}{l}
u=\ell^{\rho+n} v, v=-K\lfloor\xi\rceil_{\Delta^{r}}^{r_{n}+\tau} \\
\dot{\ell}=\mu\|\xi\|_{\Delta^{r}}^{2 \kappa}, \ell\left(t_{0}\right)=1
\end{array}\right.
$$

where $\mu \in \mathfrak{R}_{+}$is a design constant.

Remark 3.1: Compared to existing related results on universal adaptive design problem for non-parametric nonlinear systems, such as [3], [5], [16], etc., a distinguishable feature of the proposed controller is that both the proposed control law and design procedure are much simpler by totally neglecting the costly recursive calculations. More significantly, the controller design procedure is essentially detached from a recursive determination process of virtual controllers so that the step-by-step calculations of the tuning functions are also avoided. This characteristic could endow the practitioners much more convenience to implement the controller in reallife plants.

Remark 3.2: Inspired by the dynamic high gain technique in [7], [8], [9] and the facility of homogeneous system theory, we have found that if an indirect adaption law for the dynamic gain is settled, rather than directly estimating the uncertain parameters via classical tuning functions, a non-recursive adaptive synthesis approach is promising. The proposed method can be seen as an essential nonsmooth extension of the result in [8], [9]. Indeed, in a special case by setting the homogeneous degree $\tau=0$, the control law (6) could reduce to a linear universal controller of the following form:

$$
\left\{\begin{array}{l}
u=\ell^{\rho+n} v, v=-K \xi \\
\dot{\ell}=\mu\|\xi\|^{2}, \ell\left(t_{0}\right)=1
\end{array}\right.
$$

Remark 3.3: Owing to the proposed systematic nonrecursive synthesis approach, now the control gain parameter determination can be simplified as preliminary works. Similar to the method in [14], one can find a constant gain vector $K$ to render $A-B K$ Hurwitz first, and then obtain $P$ by solving LMI in (4). Therefore the value $\rho$ can be selected. Noting that the value of $\mu$ is critical to the increasing rate of $\ell$, hence, a proper choice of the parameter $\mu$ can be determined via "trial and error" method. Subsequently, the scaling gain $\ell$ will be self-tuned online from its initial value $\ell(0)=1$. Such a gain tuning mechanism can be regarded as a mediate role between adaptive and robust methods. It provides the controlled system some robustness, but the conservative selection guideline of $\ell$ in existing related robust methods, such as [13], [23], is no longer required.

An example as a demonstration of the simplicity and effectiveness of the proposed control scheme is given below.

Example 3.1: Consider the following uncertain nonlinear system:

$$
\left\{\begin{array}{l}
\dot{x}_{1}=x_{2}+\ln \left(1+\left(\theta_{1} x_{1}\right)^{2}\right) \\
\dot{x}_{2}=x_{3}+\sin \left(\theta_{2} x_{2}\right) \\
\dot{x}_{3}=u
\end{array}\right.
$$

where $\theta_{1}$ and $\theta_{2}$ are unknown parameters.

As one of the most distinguishable feature in reference to existing recursive adaptive design strategies, such as backstepping design, under the proposed framework, without going through a series of recursive determination of virtual controllers, one can design directly from system (8) of the following dynamic stabilizing controller:

$$
\left\{\begin{array}{l}
u(t)=-\ell^{\rho+3} K\left(\left\lfloor\xi_{1}\right\rceil^{4 / 7},\left\lfloor\xi_{2}\right\rceil^{2 / 3},\left\lfloor\xi_{3}\right\rceil^{4 / 5}\right)^{\top} \\
\dot{\ell}=\mu\|\xi\|_{\Delta^{r}}^{2 \kappa}, \ell(0)=1
\end{array}\right.
$$

where $\|\xi\|_{\Delta^{r}}=\sqrt{\left|\xi_{1}\right|^{2}+\left|\xi_{2}\right|^{7 / 3}+\left|\xi_{3}\right|^{14 / 5}}$. 


\section{STABILITY ANALYSIS}

In this section, we will show that global stabilization can be realized by the proposed nonsmooth adaptive controller of the form (6) under the following condition.

Assumption 4.1: There exists an unknown constant $\sigma \in \mathfrak{R}_{+}$ which is dependent on $\theta$, such that the following relation holds: $\left|f_{i}\left(\theta, \bar{x}_{i}\right)\right| \leq \sigma\left(\left|x_{1}\right|^{\frac{r_{i}+\tau}{r_{1}}}+\left|x_{2}\right|^{\frac{r_{i}+\tau}{r_{2}}}+\cdots+\left|x_{i}\right|^{\frac{r_{i}+\tau}{r_{i}}}\right), \quad i \in$ $\mathbb{N}_{1: n}$.

Remark 4.1: Assumption 4.1 can also be called as a homogeneous growth condition, which is commonly utilized for a global stabilization objective in the sense of theoretical conscientiousness, see, e.gs., [13], [15], etc. However, in real-life applications, Assumption 4.1 can also be removed by exploiting a semi-global stabilization objective, see for instance, [24], [26], etc.

The main result of this paper can be stated as follows.

Theorem 4.1: Consider the closed-loop system consisting of (1) satisfying Assumption 4.1 and the nonsmooth adaptive control scheme (6). The following conclusions hold.

i) All the signals in the closed-loop system are globally uniformly bounded.

ii) The system state will converge to the origin within a finite time.

Proof: Define a Lyapunov function candidate as $V(\xi)=$ $\left(\lfloor\xi\rceil_{\Delta^{r}}^{\gamma}\right)^{\top} P\lfloor\xi\rceil_{\Delta^{r}}^{\gamma}$. From the fact that the homogeneous degree $\tau<0$ and $\kappa>1$, we know that $V(\xi) \in \mathbb{C}^{1}$. With the dilation mapping $\Delta_{\varepsilon}^{r} \xi=\left(\varepsilon^{r_{1}} \xi_{1}, \cdots, \varepsilon^{r_{n}} \xi_{n}\right)$, one can easily verify that $V\left(\Delta_{\varepsilon}^{r} \xi\right)=\varepsilon^{2 \gamma} V(\xi)$.

Calculating the time derivative of $V(\xi)$ along system (5) gives

$$
\begin{aligned}
\dot{V}(\xi)= & \frac{\partial V(\xi)}{\partial \xi^{\top}} \ell(A \xi+B v)-\frac{\partial V(\xi)}{\partial \xi^{\top}}\left(\rho I_{n}+D\right) \xi \dot{\ell} / \ell \\
& +\frac{\partial V(\xi)}{\partial \xi^{\top}} \tilde{\digamma}(\ell, \theta, x) .
\end{aligned}
$$

To proceed, a series of estimations on the terms in (10) will be given.

Firstly, it is not difficult to obtain the following relations

$$
\left\{\begin{array}{l}
\xi_{i+1} \circ \Delta_{\varepsilon}^{r}=\varepsilon^{r_{i+1}} \xi_{i+1}=\varepsilon^{r_{i}+\tau} \xi_{i+1}, i \in \mathbb{N}_{1: n-1}, \\
\nu \circ \Delta_{\varepsilon}^{r}=-\varepsilon^{r_{n}+\tau} K\lfloor\xi\rceil_{\Delta^{r}}^{r_{n}+\tau}
\end{array}\right.
$$

which concludes that the vector field $A \xi+B v$ is $\Delta^{r}$ homogeneous of degree $\tau$. One can follow the corresponding proof in [25], [27] directly that the closed-loop system is globally finite-time stable.

In addition, noting that $\frac{\partial V(\xi)}{\partial \xi^{\top}}(A \xi+B v) \in \mathbb{H}_{\Delta^{r}}^{2 \kappa}$ and $V(\xi) \in$ $\mathbb{H}_{\Delta^{r}}^{2 \gamma}$, by applying Lemma 2.1 , there exists a constant $c_{1} \in \Re_{+}$, such that the following relation holds for $\tau \in(-\varsigma, 0)$

$$
\frac{\partial V(\xi)}{\partial \xi^{\top}}(A \xi+B v) \leq-c_{1}\|\xi\|_{\Delta^{r}}^{2 \kappa}
$$

Secondly, with Assumption 4.1 in mind, we have

$$
\begin{aligned}
& \frac{f_{i}(\cdot)}{\ell^{\rho+i-1}} \leq \frac{\sigma}{\ell^{\rho+i-1}}\left(\left|x_{1}\right|^{\frac{1+i \tau}{1}}+\left|x_{2}\right|^{\frac{1+i \tau}{1+\tau}}+\cdots+\left|x_{i}\right|^{\frac{1+i \tau}{1+(i-1) \tau}}\right) \\
= & \frac{\sigma}{\ell^{\rho+i-1}}\left(\left|\ell^{\rho} \xi_{1}\right|^{\frac{1+i \tau}{1}}+\left|\ell^{\rho+1} \xi_{2}\right|^{\frac{1+i \tau}{1+\tau}}\right. \\
& \left.+\cdots+\left|\ell^{\rho+i-1} \xi_{i}\right|^{\frac{1+i \tau}{1+(i-1) \tau}}\right) \\
= & \sigma\left(\ell^{\frac{\rho(1+i \tau)}{1}}-(\rho+i-1)\left|\xi_{1}\right|^{\frac{1+i \tau}{1}}+\ell^{(\rho+1) \frac{1+i \tau}{1+\tau}-(\rho+i-1)}\left|\xi_{2}\right|^{\frac{1+i \tau}{1+\tau}}\right. \\
& \left.+\cdots+\ell^{(\rho+i-1) \frac{1+i \tau}{1+(i-1) \tau}-(\rho+i-1)}\left|\xi_{i}\right|^{\frac{1+i \tau}{1+(i-1) \tau}}\right) .
\end{aligned}
$$

Note that $\ell \geq 1,0<\frac{1+i \tau}{1+(j-1) \tau}<1$ and $\rho>0$ which conclude that $\ell^{(\rho+j-1) \frac{1+i \tau}{1+(j-1) \tau}-(\rho+i-1)}<\ell^{0}=1$ for $j \in \mathbb{N}_{1: i}$. Hence following (12), we have

$$
\begin{aligned}
\frac{f_{i}\left(\theta, \bar{x}_{i}\right)}{\ell^{\rho+i-1}} & \leq \sigma\left(\left|\xi_{1}\right|^{\frac{1+i \tau}{1}}+\left|\xi_{2}\right|^{\frac{1+i \tau}{1+\tau}}+\cdots+\left|\xi_{i}\right|^{\frac{1+i \tau}{1+(i-1) \tau}}\right) \\
& \leq \bar{c}_{2, i} \sigma\|\xi\|_{\Delta^{r}}^{r_{i}+\tau},
\end{aligned}
$$

where $\bar{c}_{2, i} \in \Re_{+}$is a constant independent of $\ell$.

It is clear that $V(\xi) \in \mathbb{H}_{\Delta^{r}}^{2 \gamma}$. By utilizing Lemma 2.1, there exists a constant $c_{2} \in \Re_{+}$which is independent of $\ell$, such that the following relations hold

$$
\frac{\partial V(\xi)}{\partial \xi^{\top}} \tilde{\digamma}(\ell, \theta, x) \leq \sum_{i=1}^{n} \bar{c}_{2, i} \sigma\left|\frac{\partial V(\xi)}{\partial \xi_{i}}\right|\|\xi\|_{\Delta^{r}}^{r_{i}+\tau} \leq c_{2} \sigma\|\xi\|_{\Delta^{r}}^{2 \kappa} .
$$

Thirdly, from the definition of $\rho$ and $\dot{\ell} / \ell \geq 0$, it is clear that $\left(\Xi_{1} P+P \Xi_{1}\right) \rho+\Xi_{2} P+P \Xi_{2}>0$. Then with Lemma 2.1 , there must exist a constant $c_{3} \in \Re_{+}$such that the following relation holds

$$
\begin{aligned}
& \frac{\partial V(\xi)}{\partial \xi^{\top}}\left(\rho I_{n}+D\right) \xi \dot{\ell} / \ell \\
& =\left(\lfloor\xi\rceil_{\Delta^{r}}^{\gamma}\right)^{\top}\left(\left(\Xi_{1} P+P \Xi_{1}\right) \rho+\Xi_{2} P+P \Xi_{2}\right)\lfloor\xi\rceil_{\Delta^{r}}^{\gamma} \dot{\ell} / \ell \\
& \geq c_{3} \dot{\ell} / \ell\|\xi\|_{\Delta^{r}}^{2 \gamma} .
\end{aligned}
$$

Substituting (11), (14) and (15) into (10), the time derivative of $V(\xi)$ along system (5)-(6) can be reduced to the following form

$$
\begin{aligned}
\dot{V}(\xi) & \leq-c_{1} \ell\|\xi\|_{\Delta^{r}}^{2 \kappa}-c_{3} \dot{\ell} / \ell\|\xi\|_{\Delta^{r}}^{2 \gamma}+c_{2} \sigma\|\xi\|_{\Delta^{r}}^{2 \kappa} \\
& \leq-\left(c_{1} \ell-c_{2} \sigma\right)\|\xi\|_{\Delta^{r}}^{2 \kappa} .
\end{aligned}
$$

i) In what follows, motivated by [9], we will use contradiction arguments to prove that there will be no finite escape time of the trajectory $\left(\xi^{\top}, \ell\right)^{\top}$ starting from $t_{0}$. If the above statement is not true, there must exist a maximal time interval $\left[\mathrm{t}_{0}, t_{f}\right)$ where $t_{f}>t_{0}$, such that $\left(\xi^{\top}, \ell\right)^{\top}$ are well defined and $\lim _{t \rightarrow t_{f}}\left\|\left(\xi^{\top}, \ell\right)^{\top}\right\|=+\infty$.

Firstly, we show that $\ell$ cannot escape at $t=t_{f}$. Otherwise, the relation $\lim _{t \rightarrow t_{f}} \ell=+\infty$ holds. Noting that $c_{1}, c_{2}, \sigma$ are all bounded, hence there exists a time instant $t_{1}$ satisfying $t_{0}<$ $t_{1}<t_{f}$, such that $c_{1} \ell-c_{2} \sigma \geq 1, t \in\left[t_{1}, t_{f}\right]$. This relation could lead to a fact that $\dot{V}(\xi) \leq-\|\xi\|_{\Delta^{r}}^{2 \kappa}, t \in\left[t_{1}, t_{f}\right]$.

Therefore, with the update law (6), it is straightforward that

$$
\begin{aligned}
+\infty & =\ell\left(t_{f}\right)-\ell\left(t_{1}\right)=\int_{t_{1}}^{t_{f}} \dot{\ell}(s) d s=\int_{t_{1}}^{t_{f}} \mu\|\xi(s)\|_{\Delta^{r}}^{2 \kappa} d s \\
& \leq \mu V\left(\xi\left(t_{1}\right)\right)<+\infty,
\end{aligned}
$$


which is clearly a contradiction. Then one could claim that $\ell$ is well defined in the time interval $\left[t_{0}, t_{f}\right]$.

Secondly, we show that $\xi$ cannot escape at $t=t_{f}$. Integrating both sides of (16) over the time interval $\left[t_{0}, t\right]$ yields

$$
\begin{aligned}
V(\xi(t))-V\left(\xi\left(t_{0}\right)\right) \leq & -\int_{t_{0}}^{t} c_{1} \ell(s)\|\xi(s)\|_{\Delta^{r}}^{2 \kappa} d s \\
& +\int_{t_{0}}^{t} c_{2} \sigma\|\xi(s)\|_{\Delta^{r}}^{2 \kappa} d s \\
\leq & -\int_{t_{0}}^{t} \frac{c_{1}}{\mu} \ell(s) \dot{\ell}(s) d s+\int_{t_{0}}^{t} \frac{c_{2} \sigma}{\mu} \dot{\ell}(s) d s \\
\leq & -\frac{c_{1}}{2 \mu}\left(\ell^{2}(t)-1\right)+\frac{c_{2} \sigma}{\mu}(\ell(t)-1) .
\end{aligned}
$$

Hence one could deduce the relation that

$$
V\left(\xi\left(t_{f}\right)\right) \leq V\left(\xi\left(t_{0}\right)\right)-\frac{c_{1}}{2 \mu}\left(\ell^{2}\left(t_{f}\right)-1\right)+\frac{c_{2} \sigma}{\mu}\left(\ell\left(t_{f}\right)-1\right) .
$$

From the fact that $\ell\left(t_{f}\right)$ is bounded, it is obvious that $V\left(\xi\left(t_{f}\right)\right)$ is also bounded. With Lemma 2.1, it is clear that there exists a constant $c_{4} \in \Re_{+}$such that $\left\|\xi\left(t_{f}\right)\right\|_{\Delta^{r}} \leq$ $c_{4} V^{\frac{1}{2 \gamma}}\left(\xi\left(t_{f}\right)\right)$, which implies that $\xi\left(t_{f}\right)$ is bounded. This fact clearly contradicts the finite escape time claim.

In a conclusion, we have already proved that $\left(\xi^{\top}, \ell\right)^{\top}$ is well defined and globally uniformly bounded for $t \in\left[t_{0}, \infty\right)$.

ii) Using the boundness of $\dot{\xi}$ and $\xi$ for $t \in\left[t_{0}, \infty\right)$. it can be straightforwardly deduced from Barbalat's Lemma that $\xi \rightarrow$ $0, t \rightarrow \infty$. In addition, by recalling from the expression (6) that $\ell$ is monotone increasing and $c_{1}, c_{2}, \sigma$ are bounded, we know there must exist a time instant $T_{1} \geq t_{0}$ such that $c_{1} \ell-c_{2} \sigma>$ $0, \forall t \geq T_{1}$. Note that with Lemma 2.1, $\|\xi\|_{\Delta^{r}}^{2 \kappa} \geq c_{5} V^{\frac{2 \kappa}{2 \kappa-\tau}}(\xi)$ with $c_{5}$ being a positive constant. Then with (16) in mind, the following relation holds

$$
\dot{V}(\xi)+c_{5}\left(c_{1} \ell-c_{2} \sigma\right) V(\xi)^{\frac{2 \kappa}{2 \kappa-\tau}} \leq 0, t \geq T_{1} .
$$

Based on (18), it can be deduced straightforwardly from Lemma 2.2 that the there exists a finite time $T_{2} \geq T_{1}$, such that $\xi(t)=0, \forall t \geq T_{2}$. This completes the proof of Theorem 4.1 .

Remark 4.2: As implied by the proof, the closed-loop system stability is directly dependent on a sufficiently large scaling gain $\ell$ which is subject to a simple self-tuning update law in (6). Indeed, it can be concluded from the initial value $\ell\left(t_{0}\right)=1$ that the closed-loop system could possibly be unstable at the initial stage. However, $\ell$ is made monotone increasing as $\dot{\ell}(t) \geq 0$, which is illustrated in the simulation of Example 3.1 later on. After a short period, $\ell(t)$ can be sufficiently large to render the closed-loop system stable.

\section{Numerical Simulation}

Consider Example 3.1 in the case when $\theta_{1}=0.2 \sin (t)$ and $\theta_{2}=0.1$ are unknown parameters to the designer.

Denoting $\sigma=\max \left\{2\left|\theta_{1}\right|^{6 / 7},\left|\theta_{2}\right|^{5 / 6}\right\}$, one can verify the following relations $\left|f_{1}\left(\theta, x_{1}\right)\right|=\left|\ln \left(1+\left(\theta_{1} x_{1}\right)^{2}\right)\right| \leq 2\left|\theta_{1}\right|^{6 / 7}$. $\left|x_{1}\right|^{6 / 7} \leq \sigma\left|x_{1}\right|^{6 / 7},\left|f_{2}\left(\theta, x_{2}\right)\right|=\left|\sin \left(\theta_{2} x_{2}\right)\right| \leq \sigma\left|x_{2}\right|^{5 / 6}$. Thus system (8) satisfies Assumption 4.1 with $\tau=-1 / 7, r_{1}=$ $1, r_{2}=6 / 7, r_{3}=5 / 7$.

Before doing simulation, $K$ is firstly selected as in $[1,3,3]$ to render its companion matrix $A-B K$ Hurwitz which is

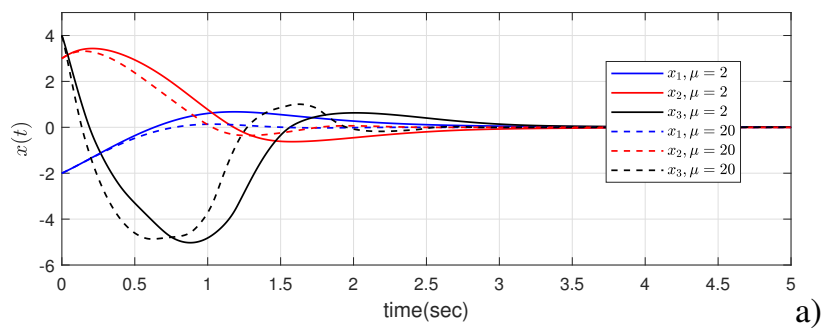

a)

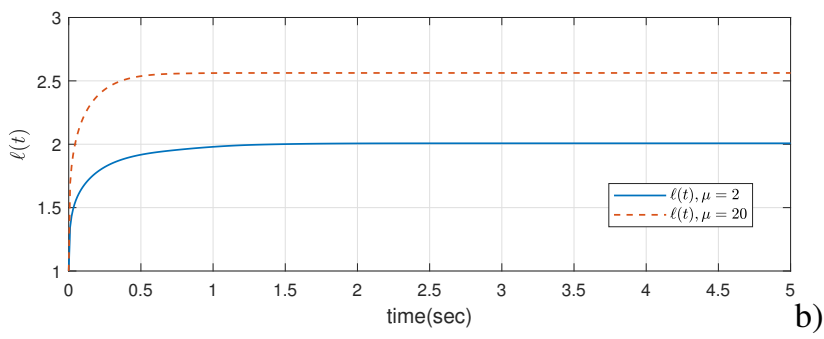

b)

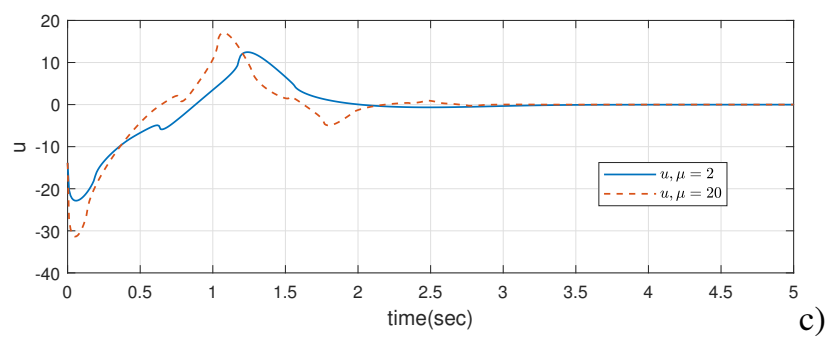

Fig. 1. Response curves under the proposed controller: a). system states; b). dynamic gain $\ell$; c). time history of the input.

quite easy, and by solving $(A-B K)^{\top} P+P(A-B K) \leq-I$, one can determine a proper matrix $P=\left[\begin{array}{ccc}2.31 & 1.94 & 0.50 \\ 1.94 & 3.25 & 0.81 \\ 0.50 & 0.81 & 0.44\end{array}\right]$. Secondly, set $\kappa=1.2$. Calculating the smallest eigenvalue of $\Xi_{1} P+P \Xi_{1}$ gives 0.76 , then the design parameter $\rho$ can be set as 2. Aiming to show impact on the control performance from the design parameter $\mu$, we select two different values in the simulation, i.e., $\mu=2$ and 20 , respectively. To conduct the simulation, the initial values are set as $x(0)=[-2,3,4]$.

It can be observed from Fig. 1. a) and b) that all the signals in the closed-loop system (8)-(9) are uniformly bounded and the trajectories of the states converge to the origin in a satisfactory control performance. The time history of the control signal is shown in Fig. 1. c). Note that a larger $\mu$ could require a larger control effort, but lead to a faster response.

\section{Conclusions}

In this paper, a simple nonrecursive nonsmooth adaptive stabilizing control design methodology for a class of nonlinear systems with the presence of non-parametric uncertainties is proposed. Distinguishable from popular methods including adaptive backstepping and adding a power integrator, etc., a simplest non-nested controller form with an explicit one-step gain tuning mechanism is obtained based on homogeneous system theory. As a result, the control implementation could be more promising with guaranteed control performance. The stability analysis is also largely simplified from prior works. 
Future works could focus on more general nonlinear systems subject to mismatched disturbances and more general nonlinear systems, etc.

\section{REFERENCES}

[1] H. K. Khalil, Nonlinear Systems. New York: Macmillan Publishing Company, 1992.

[2] M. Krstic, I. Kanellakopoulos, and P. V. Kokotovic, Nonlinear and Adaptive Control Design. John Wiley, 1995.

[3] W. Lin and C. Qian, "Adaptive control of nonlinearly parameterized systems: a nonsmooth feedback framework," IEEE Transactions on Automatic Control, vol. 47, no. 5, pp. 757-774, 2002.

[4] Y. Hong, J. Wang, and D. Cheng, "Adaptive finite-time control of nonlinear systems with parametric uncertainty," IEEE Transactions on Automatic Control, vol. 51, no. 5, pp. 858-862, 2006.

[5] J. Huang, C. Wen, W. Wang, and Y. Song, "Design of adaptive finitetime controllers for nonlinear uncertain systems based on given transient specifications," Automatica, vol. 69, pp. 395-404, 2016.

[6] J. Yang, Z. Ding, S. Li, and C. Zhang, "Continuous finite-time output regulation of nonlinear systems with unmatched time-varying disturbances," IEEE Control Systems Letters, vol. 2, no. 1, pp. 97-102, 2018

[7] P. Krishnamurthy and F. Khorrami, "Dynamic high-gain scaling: state and output feedback with application to systems with iss appended dynamics driven by all states," IEEE Transactions on Automatic Control, vol. 49, no. 12, pp. 2219-2239, 2004.

[8] L. Praly and Z. Jiang, "Linear output feedback with dynamic high gain for nonlinear systems," Systems and Control Letters, vol. 53, pp. 107116, Feb. 2004.

[9] H. Lei and W. Lin, "Universal adaptive control of nonlinear systems with unknown growth rate by output feedback," Automatica, vol. 42, no. 10 , pp. 1783-1789, 2006

[10] M. Koo, H. Choi, and J. Lim, "Universal control of nonlinear systems with unknown nonlinearity and growth rate by adaptive output feedback," Automatica, vol. 47, no. 10, pp. 2211-2217, 2011.

[11] S. P. Bhat and D. S. Bernstein, "Continuous finite-time stabilization of the translational and rotational double integrators," IEEE Transactions on Automatic Control, vol. 43, no. 5, pp. 678-682, 1998.

[12] X. Huang, W. Lin, and B. Yang, "Global finite-time stabilization of a class of uncertain nonlinear systems," Automatica, vol. 41, no. 5, pp. 881-888, 2005.

[13] J. Polendo and C. Qian, "A generalized homogeneous approach for global stabilization of inherently nonlinear systems via output feedback," International Journal of Robust and Nonlinear Control, vol. 17, no. 7, pp. 605-629, 2007.

[14] X. Zhang, G. Feng, and Y. Sun, "Finite-time stabilization by state feedback control for a class of time-varying nonlinear systems," Automatica, vol. 48, no. 3, pp. 499-504, 2012.

[15] Z. Sun, L. Xue, and K. Zhang, "A new approach to finite-time adaptive stabilization of high-order uncertain nonlinear system," Automatica, vol. 58, pp. 60-66, 2015.

[16] Z. Sun, T. Li, and S. Yang, "A unified time-varying feedback approach and its applications in adaptive stabilization of high-order uncertain nonlinear systems," Automatica, vol. 70, pp. 249-257, 2016.

[17] Z. Liu and Y. Wu, "Universal strategies to explicit adaptive control of nonlinear time-delay systems with different structures," Automatica, vol. 89, pp. 151-159, 2018.

[18] T. Li, J. Yang, S. Li, C. Wen, and C. Zhang, "Global adaptive finite-time stabilization of uncertain time-varying p-normal nonlinear systems without homogeneous growth nonlinearity restriction," IEEE Transactions on Automatic Control, vol. 64, no. 11, pp. 4637-4644, 2019.

[19] S. Zhou and Y. Song, "Neuroadaptive control design for pure-feedback nonlinear systems: A one-step design approach," IEEE Transactions on Neural Networks and Learning Systems, vol. 31, no. 9, pp. 3389-3399, 2019.

[20] X. Huang, Y. Song, and C. Wen, "Output feedback control for constrained pure-feedback systems: A non-recursive and transformational observer based approach," Automatica, vol. 113, p. 108789, 2020.

[21] J. Gao, L. Wang, R. Gao, and J. Huang, "Adaptive control of uncertain underactuated cranes with a non-recursive control scheme," Journal of the Franklin Institute, vol. 356, no. 18, pp. 11305-11317, 2019.

[22] C. Zhang and C. Wen, "A non-recursive $c 1$ adaptive stabilization methodology for nonlinearly parameterized uncertain nonlinear systems," Journal of the Franklin Institute, vol. 355, no. 12, pp. 5099-5113, 2018.
[23] C. Zhang, J. Yang, and C. Wen, "Global stabilisation for a class of uncertain non-linear systems: a novel non-recursive design framework," Journal of Control and Decision, vol. 4, no. 2, pp. 57-69, 2017.

[24] C. Zhang, J. Yang, Y. Yan, L. Fridman, and S. Li, "Semi-global finitetime trajectory tracking realization for disturbed nonlinear systems via higher-order sliding modes," IEEE Transactions on Automatic Control, vol. 60 , no. 5, pp. 2185-2191, 2020.

[25] S. Bhat and D. Bernstein, "Geometric homogeneity with applications to finite-time stability," Mathematics of Control, Signals and Systems, vol. 17, no. 2, pp. 101-127, 2005.

[26] Y. Yan, C. Zhang, A. Narayan, J. Yang, S. Li, and H. Yu, "Generalized dynamic predictive control for nonparametric uncertain systems with application to series elastic actuators," IEEE Transactions on Industrial Informatics, vol. 14, no. 11, pp. 4829-4840, 2018.

[27] W. Perruquetti, T. Floquet, and E. Moulay, "Finite-time observers: Application to secure communication," IEEE Transactions on Automatic Control, vol. 53, no. 1, pp. 356 - 360, 2008. 